\title{
SYMPTOMS OF POST-STROKE DEPRESSION
}

\author{
D. Komsiyska* \\ ${ }^{1}$ Department of Neurology and Psychiatry, Medical Faculty, Trakia University Hospital, \\ Stara Zagora, Bulgaria
}

\begin{abstract}
The purpose of this manuscript is to present our research on post-stroke depression and compare them to previous observations. Depression is a very common among disorders found in stroke patients, thus there is a high necessity in its research. We have used a contingent of 93 patients in the acute phase after stroke, which were treated at the Neurological Clinic to the University Hospital "Prof. Dr. Stoyan Kirkovich" in Stara Zagora for the period 2015 - 2016. We tested them with The Hamilton Depression Rating Scale (HAM-D-17) and analyzed the results with Statistica, version 10 (StatSoft, Inc.). We found high occurrence of depression in stroke patients. We tested for its severity and for its different symptoms in order to compare them to other studies and discover which are most common in post-stroke depression.

As a conclusion, we summarize our results that among our contingent there are predominantly mild and moderate depressive disorders with reduced mood, sadness, increased psychic and somatic anxiety, insomnia, somatic complaints and superfluous hypochondriac thoughts.
\end{abstract}

Key words: Superfluous thoughts, energy potential, psychomotor retardation, anxiety, sleep.

\section{INTRODUCTION}

According to various researchers, the incidence of affective disorders after stroke reaches 30 $60 \%$ (1). Depressive disorders are the most common psychiatric problems after a stroke (2). In one study (3) $16.4 \%$ of patients were diagnosed with post-stroke depression, where $5.8 \%$ had major depression, $8.5 \%$ - lower depression and $2.1 \%$ - dysthymia. Post-stroke depression is associated with higher mortality, especially among younger patients. It is particularly important that patients with poststroke depressive have a 3-4 times higher mortality than nondepressive patients (4). Poststroke depression has an early onset in most cases. In the studies conducted by Andersen et al., 1994 (5) and Aben et al., 2003 (6) about $50 \%$ of the cases of post-stroke depression were diagnosed in the first month after stroke.

\footnotetext{
*Correspondence to: Detelina Yordanova Komsiyska, Department of Neurology and Psychiatry, Medical Faculty, Trakia University, Stara Zagora, Bulgaria, E-mail:

detelinakomsiiska@abv.bg, tel.: +359898277673 / 042648877
}

Paolucci et al., 2005 (7) reported that for more than $60 \%$ of the patients who developed depression, mood disorders occurred in the first six weeks and this rate increased to $80 \%$ during the first three months of stroke. The duration of depression varies widely (8). In the study of Estrom et al. (9), $60 \%$ of the patients with early depression recover in one year. Similarly, according to a study by Burvill et al. (10), $41 \%$ of the patients who were depressed 4 months after stroke remained depressed in the next 12 months as well. Based on the results of Estrom et al. (9), there appears to be a high risk of chronic depression if the patient did not recover in the first year after the stroke. The pathogenesis of stroke-induced psychopathological conditions is always complex and has not been sufficiently studied to date. The physical impairment and depression, are the link between neuropsychological sequelae and other diseases $(11,12)$. For many years, only the psychogenic response to the disability was believed to be the major cause of post-stroke depression (10). Subsequently, psychosocial factors have begun to attach some importance 
to endogenous factors (individual personality and hereditary predisposition) (13).

The issue of the etiology of post-stroke depression is explored by two main approaches: some suggest that post-stroke depression is caused by brain damage itself. On the contrary, others suggest that this is a psychological response to disability or loss. Many previously described discoveries can be considered as evidence of both the physiological and psychosocial mechanisms of post-stroke depression. The two approaches are not mutually exclusive, but instead describe post-stroke depression as a complex and multifactorial disease with interaction between physiological factors and environmental factors (14).

Particular interest arises in the mutual relation of the damages of certain brain structures and the neurotransmitter's disturbed balance $(15,16)$. Researchers suggest the existence of neuroanatomic and pathophysiological conditions for the development of affective disorders involving neurochemical changes in the post-stroke period (17). At the heart of the pathogenetic mechanisms of development of both anxiety and depression there is dysfunction of the neurotransmitter systems of the central nervous system, of which the most importance is attributed to the serotonin system.

It is believed that disruption of the pathways of biogenic amines caused by the stroke, that cause disorders in the synthesis and metabolism of monoamine transmiters, may play an etiological role in post-stroke depression. It is believed that endogenous depression is caused by neurotransmitter changes. The deficiency of the monoamine transmitters dopamine, serotonin and noradrenaline, or damage to the entire system of monoamine neurotransmitters in different brain chains may cause depression. In the study of post-stroke depression and endogenous depression, interest is shifted by the monoamine neurotransmitters themselves to their receptors and to the molecular events that these receptors unlock. These include transduction of the flow signal and regulation of gene expression. For example, it is assumed that, under stress, the gene for brain-derived neurotrophic factors may be suppressed.

A new hypothesis for post-stroke depression is the increased production of proinflammatory cytokines and the importance of specific high- risk genes. The time elapsed from stroke becomes particularly important when poststroke depression is seen as a natural psychological reaction to stroke and its consequences (18). Denial is typical in the acute phase followed by anger or frustration and weeks later - by depression. Reception increases with time and usually can not be reached earlier than one year after the loss. Among patients with stroke there are at least two additional factors that complicate this process. First, stroke patients become aware of their disabilities gradually. Typically, this occurs in different ways with different symptoms. Secondly, injuries caused by stroke improve with spontaneous recovery and rehabilitation. Some authors associate both psychological and somatic symptoms with post-stroke depression and support the use of Diagnostic and Statistical Manual criteria regardless of the nature or origin of the symptoms (19). The few studies, where both the temporal aspect and the various symptoms are addressed, give conflicting results.

\section{METHODS}

The study contingent was 93 stroke patients, of which 59 men (39-83 years old) and 34 women (56-87 years old), with mean age 66.8 years. Patients were treated at the Neurological Clinic to the University Hospital "Prof. Dr. Stoyan Kirkovich" in Stara Zagora for the period 2015 - 2016, meeting the criteria set by the protocol. The patients have been informed in advance of the objectives, the tasks and the methods of the study and have provided written consent for participation.

We served with the Hamilton Depression Rating Scale (HAM-D-17) to assess the severity of depressive disorder according to the tenth version of the International Statistical Classification of Diseases and Related Health Problems (ICD-10) criteria;

Statistical method - statistical analyzes were performed using the statistical program package for Windows 7 - Statistica version 10 (StatSoft, Inc.).

\section{RESULTS}

Of all the patients surveyed by us, 60 (64.5\%) had depressive disorder. The severity of the diagnosed depressive states is objectively evaluated by HAM-D-17 as follows: from 8 to 13 points - a slight degree, from 14 to 18 points - medium grade, from 19 to 22 points moderately severe grade and 23 to 54 points severe grade. Patients with mild depressive disorder episode, 22 (36.6\%), and moderate 
depressive episode, 18 (30\%), prevail. Fewer are patients with moderately severe depressive episode, $10(16.6 \%)$, and severe depressive episode, $10(16.6 \%)$. We have not observed psychotic symptoms within the depressive disorder. The majority of our patients have insight (self-perceived illness) - 35 (58.3\%). We found decreased mood (sadness, despair, helplessness, futility) as a major symptom of the depressive disorder in $92(87 \%)$ of the depressive patients. As a whole $19(31.7 \%)$ of the patients spontaneously verbalised, 13 $(21.7 \%)$ only on asking, $12(20 \%)$ expressed spontaneous verbal and non-verbal expression and only in $8(13.3 \%)$ of the patients nonverbal behavior (face, posture, voice) was observed.

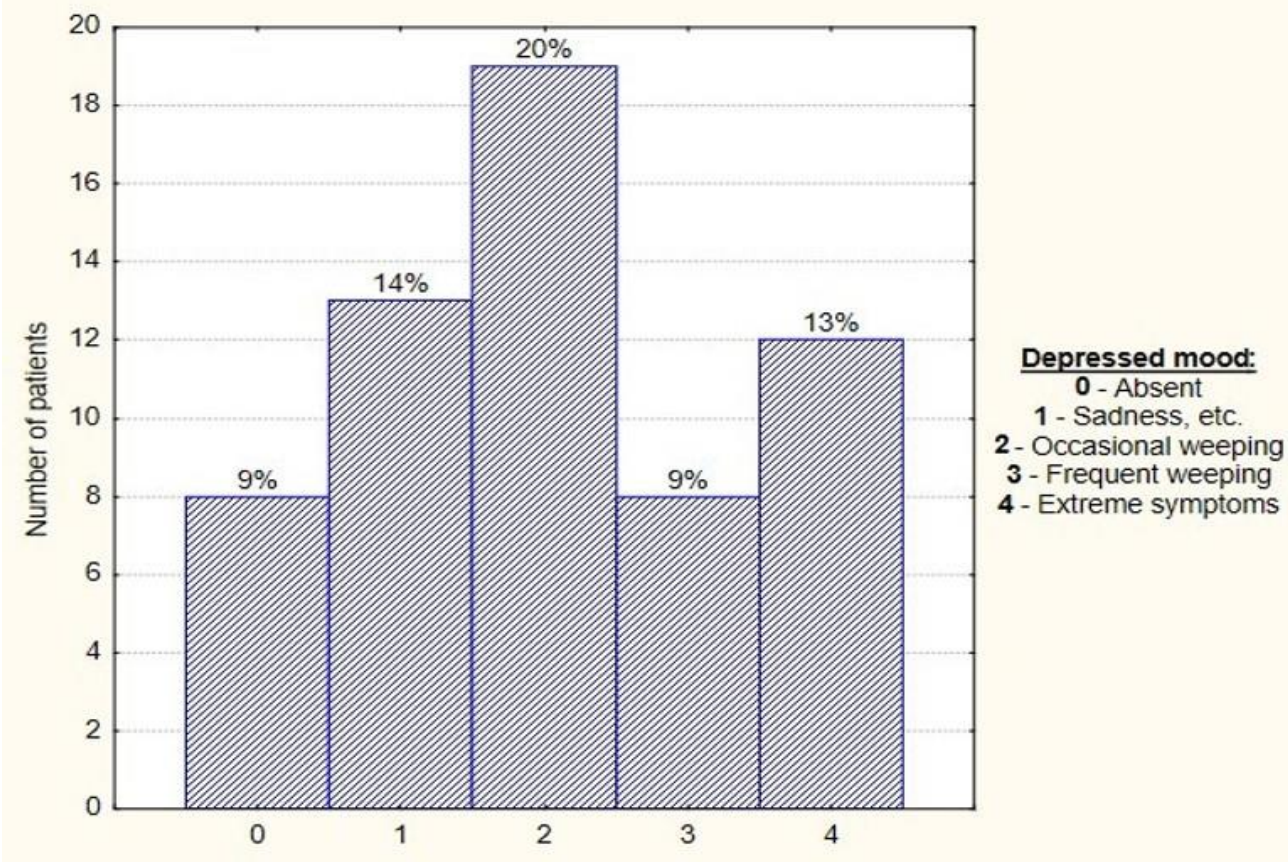

Figure 1. Changes in mood in patients with depressive disorder

\section{Depressed mood}

Gainotti et al., (20) suggest that patients with post-stroke depression are more likely to manifest catastrophic reactions, agitation and day-night mood changes than patients with idiopathic depression, although these findings have not been confirmed by other studies. Depressive thoughts of superfluous nature in depressive syndrome were observed in their different varieties. Superfluous thoughts of inferiority were observed in $6(10 \%)$ of depressed patients, while superfluous thoughts of guilt at $14(23.3 \%)$. Superfluous thoughts of lack of perspective reported only $4(6.7 \%)$ of depressed patients, while superfluous thoughts of hopelessness shared $2(3.3 \%)$. In one $(1.7 \%)$ of the depressed patients there were suicidal ideas and one $(1.7 \%)$ had attempted suicide. Higher suicidal risk is reported by Tang et al (21) $-6.2 \%$ of post-stroke patients. Kishi et al (22) reported that suicidal risk increases with time spent after a stroke. According to this study the presence of apathy is a predictor for the emergence of suicidal ideas. A massive study by Eriksson et al (23) reported that patients with low education or income, living alone, male, young, with severe stroke and the presence of post-depressive disorder are more prone to suicide. Garden et al (24), evaluating suicidal risk after stroke, point that suicidal risk factors are the presence of depression, severe insomnia, chronic disease, and organic brain syndrome.

Energy potential is an indicator, which is given special attention to patients with stroke. Elevated tiredness may be an expression of neurosis symptomatology within the psychoorganic stroke syndrome. The decline in its activity productivity, feelings of fatigue, and weakness symptoms of the depressive disorder in post-stroke patients are an important part of the Clinical Characteristics of the depressive disorder.

Only $5(8 \%)$ of patients with comorbid depressive disorder did not report reduction of energy potential. Increased fatigue reported 22 $(36.7 \%)$ of the patients, loss of interest -21 $(35 \%)$. In $10(16.7 \%)$ there was a decrease in the time spent on work or other activities and in two $(3.3 \%)$ - complete stopping of work. 


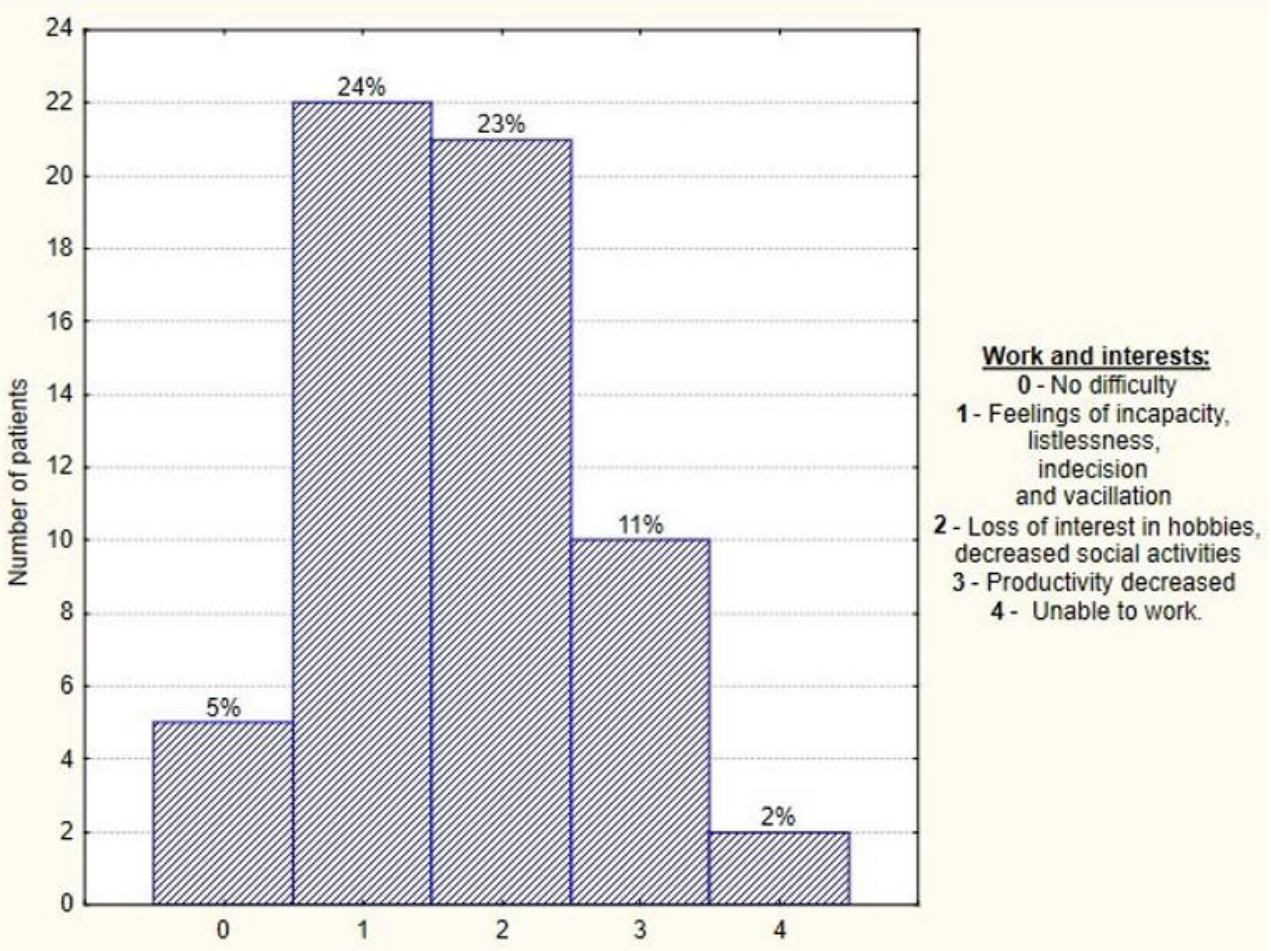

Figure 2. Work and interests in patients with depression

\section{Work and interests}

Of patients without depression in 19 (57.6\%) there was no change in working capacity, 14 $(42.4 \%)$ experienced increased tiredness and none of them suffered from more serious grades fatigue.

Psychomotor retardation is an indicator, whose evaluation includes a number of cognitive symptoms (concentration, active attention, memory, executive functions). It can also be found in the psycho-organic syndrome and the depressive disorder syndrome. Comorbidity between the two conditions could increase the frequency and the severity of psychomotor retardation. In $28(46.7 \%)$ of patients with strokes and depressive disorder account is taken of the presence of psychomotor retardation, with $22(36.7 \%)$ of them being mildly expressed, 5 (8.3\%) - notably, and in 1 $(1.7 \%)$ the interview was difficult. Lipsey et al (25) find that the presence of retardation psychomotor retardation in patients with poststroke depression, some of the differentiators symptoms of idiopathic depressed patients, lead to complaints from more anhedonia and difficult concentration.

Of patients without depression, $28(84.85 \%)$ did not suffer from psychomotor retardation, 5 $(15.15 \%)$ suffered from a slight delay during the interview and none of them suffered from more serious degrees of psychomotor retardation.

Anxiety is common within the depressive disorder and, on the other hand, comorbid depressive disorder intensifies the present increased anxiety, which has a multifactorial genesis during strokes. Increased mental anxiety within the depressive syndrome was found in $52(86.6 \%)$ of post-stroke patients with comorbid depressive disorder. In 8 $(13.3 \%)$ of the patients it was expressed in increased internal tension and irritability, and in $39(65 \%)$ - in increased concern for unimportant things. In $5(8.3 \%)$ increased anxiety was expressed spontaneously through facial expression or speech.

\section{Anxiety - psychic}

Somatic anxiety, expressed in anxiety-caused gastrointestinal, cardiovascular or respiratory symptoms, was found in $47(78.4 \%)$ of depressed patients with strokes, and most commonly somatic anxiety was modestly expressed - in 28 (46.7\%). Weak expression was observed in $10(16.7 \%)$, and strong expression - in 9 (15\%). During the study events of different in degree agitation was observed in $14(23.3 \%)$ of patients.

Of patients without depression $24(72.7 \%)$ did not suffer from somatic anxiety, $5(15.15 \%)$ 
had poorly expressed somatic anxiety, 4 $(12.1 \%)$ had moderate symptoms and none with strong or extreme degrees. Of patients without depression 29 (87.9\%) did not have gastrointestinal complaints, 3 (9.1\%) experienced loss of appetite, but were fed
KOMSIYSKA D.

without encouragement from staff and experienced severe sensitivity in the abdomen and only $1(3 \%)$ experienced difficulty feeding without the urge of staff, wanted or needed laxatives or gastrointestinal medications.

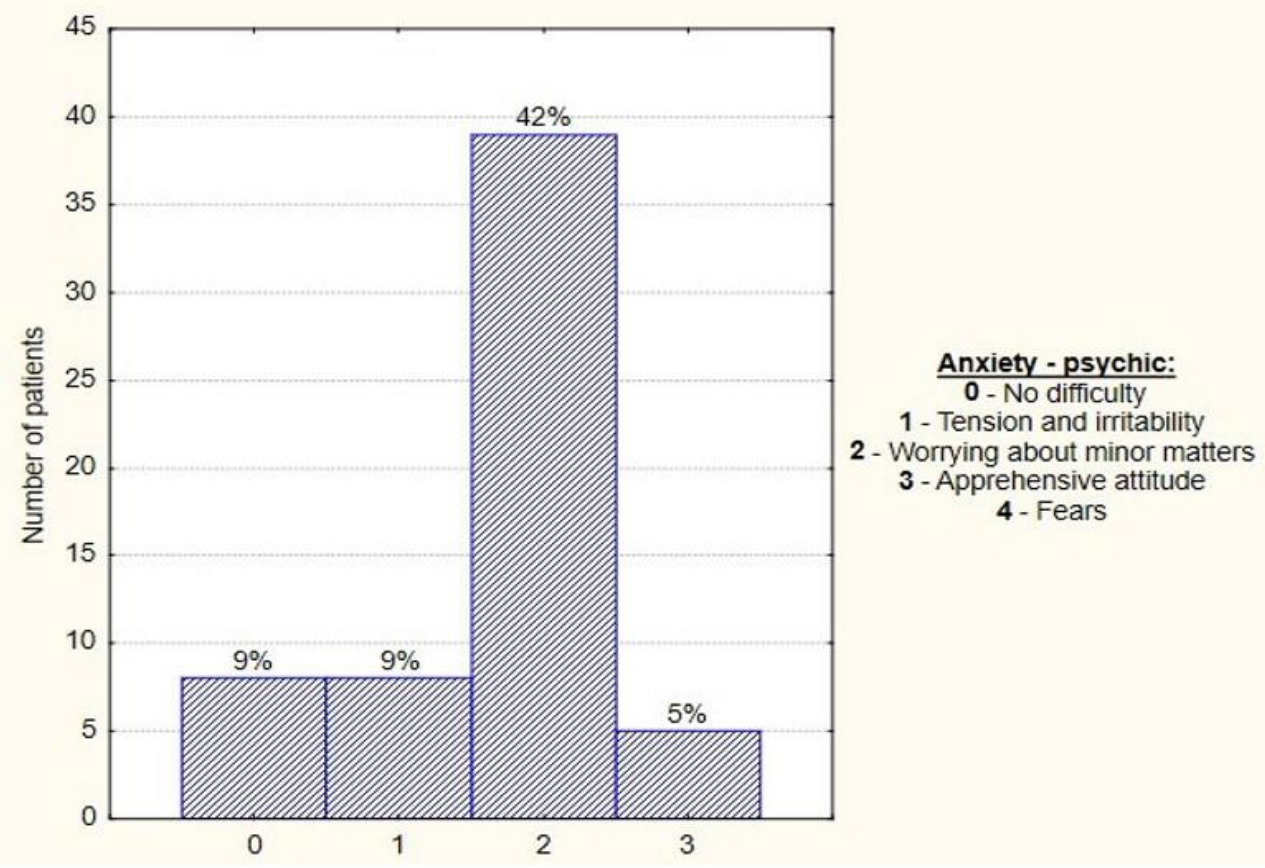

Figure 3. Psychic anxiety in patients with depression

Sleep disturbances were expressed the most from somatic depressive symptoms. Problems with sleeping reported $31(51.7 \%)$ patients with strokes and comorbid depressive disorder, with $22(36.7 \%)$ having sleeping difficulties every night. In $40(66.6 \%)$ of the post-stroke depressive patients there were disruptions in the continuity of sleep, and $42(70 \%)$ had an early wakening.

In $27(81.8 \%)$ of patients without depression early insomnia was missing, $2(6.1 \%)$ had occasional sleeping problems and $4(12.1 \%)$ had difficulties every night. In 30 (90.9\%) of patients without depression medium insomnia was absent, 1 (3\%) suffered from uneasiness and anxiety at night and $2(6.1 \%)$ woke up at night. In $28(84.85 \%)$ of patients without depression late insomnia was absent, 3 (9.1\%) complained from getting out of bed, but with a subsequent return to sleep, and 2 (6.1\%) suffered from inability to re-fall asleep.

Reduction or loss of appetite reported 25 (41.6\%) of patients, with only 19 (31.6\%) reporting a reduction in body weight, while genital symptoms (libido disturbance, menstrual disorders) were usually absent or were not found in our patients. Only in 10 $(16.7 \%)$ they were poorly expressed and for one $(1.7 \%)$ they were a serious problem.

Of general somatic symptoms (burden in the limbs, back or head, back pain, head or muscles, loss of energy and easy fatigue) suffered most patients $(52(86.7 \%))$ with poststroke depression. While vegetative symptoms (such as changes in sleep, appetite, and sexual impulse) as well as fatigue are common symptoms of depressive and (acute or chronic) neurological disorders, including stroke, the presence of post-stroke depression may reinforce the severity of vegetative symptoms. Our results are shared by a study of Fedoroff et al. (19), in which vegetative symptoms, including sleep disorders, libido and energy level, were significantly more common in depressed than in non-depressed patients with stroke. From patients without depression 19 (57.6\%) had no general somatic complaints, 12 (36.4\%) complained about a burden in the limbs, back or head, back pain, head or muscles, loss of energy and easy fatigue and 2 $(6.1 \%)$ of patients with depression shared about clearly outlined symptoms. 


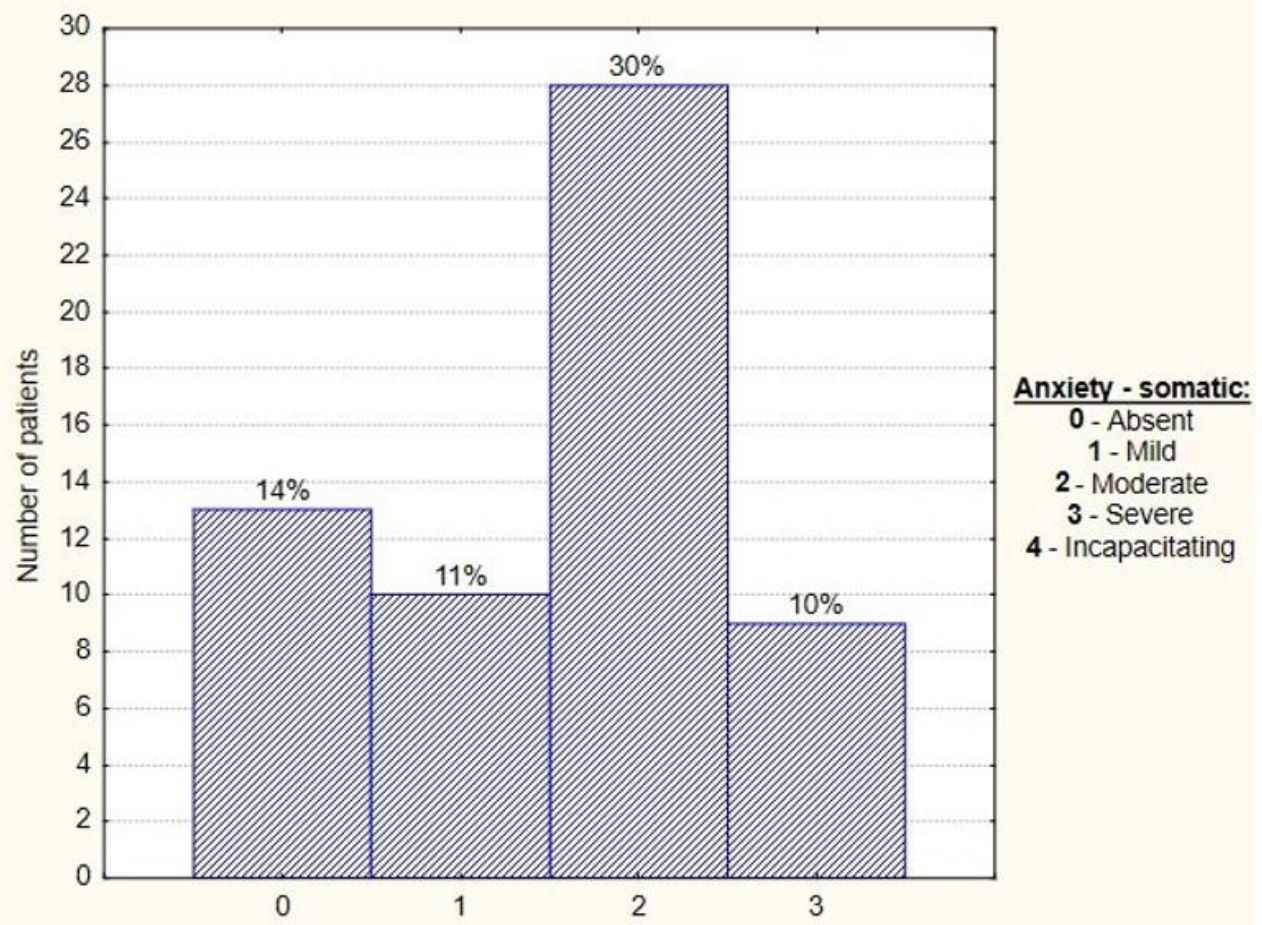

Figure 4. Somatic anxieties in patients with depression

\section{Anxiety - somatic}

Excessive hypochondrial thoughts were observed in $47(78.3 \%)$ of patients with depression. In $9(15 \%)$ body weight selfabsorption was present, in 27 (45\%) there was excessive concern for the health and in 11 (18.3\%) frequent complaints, calls for help, etc. were observed. No trend to develop hypochondriacal delusions, which is a common symptom for involutive depression.

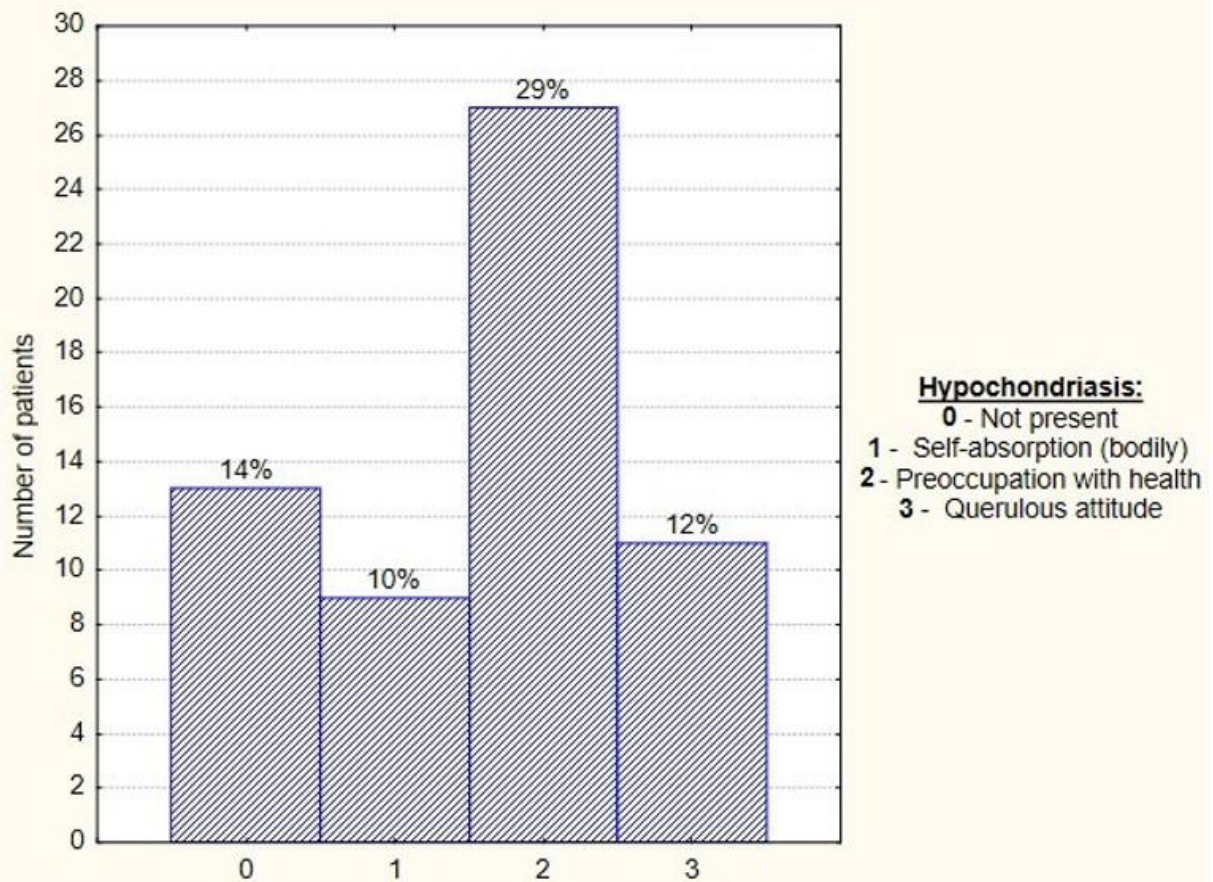

Figure 5. Hypochondriasis in patients with depression

\section{Hypochondriasis}

In its predominant part the comorbid depressive disorder of the patients in our study was mild and moderately severe. Against the background of decreased mood $(81.6 \%)$ and sadness $(73.3 \%)$, increased mental $(86.6 \%)$ and somatic (78.4\%) anxiety, insomnia, predominantly with early morning wakening, there was low level of expression of the common for endogenous depression excessive thoughts of inferiority, guilt, lack of perspective and hopelessness. 
Suicidal thoughts shared $1.7 \%$ of depressive patients, with only one having a suicidal attempt in the past. Most of the patients with post-stroke depression - 52 (86.7\%), were involved with somatic complaints (burden in the limbs, back or head, back pain, head or muscles, loss of energy and easy fatigue) and their consequences - superfluous hypochondrial thoughts $(78.3 \%)$.

Using factor analysis Savina, 2016 (26) outlines the limits of the depressive syndrome after a stroke which has a mosaic structure and includes the following depressive symptoms: grief $(87.3 \%)$, anhedonia (55.1\%), depressive apathy $(25.4 \%)$, hopelessness $(25.4 \%)$ and dysphoria (50.8\%), and also the presence of vegetative disorders. The syndrome it describes does not completely comply with international criteria classifications: dysphoria often enters it, as well as asthenous-like disorders such as weakness and tiredness, that add more weight towards organic spectrum disorders.

Expressed concern about body health discusses Arnaudova (27) in late-onset depressions with its frequent masking behind somatic symptoms, which complicates early diagnosis and treatment of these conditions. However, there is no doubt that somatic symptoms in the elderly may not be associated with depression, which would lead to an underestimation of existing real somatic problems. Depression in adults are to a large extent "somatic", that is to say somatic symptoms overwhelm the rest.

According to Rabi-Zikic et al (28) non-specific somatic symptoms do not interfere to diagnosing post-stroke depression and can be very important for evaluation of post-stroke depression, as opposed to the general opinion. So far as the clinical manifestations of depressive stroke disorder mostly have somatic nature with predominance of anxiety disorders (29), it is necessary to take into account high sensitivity of patients even to an insignificant increase of the body discomfort from poststroke depression.

Socio-cultural traditions of the Bulgarian people also contribute to the fact that the patients more often and willingly report to the treating physicians complaints of somatic nature, considering emotional discomfort as unimportant and not deserving discussion. In one study (30) the overall symptom score of patients diagnosed with major depression without clinical comorbidity, is significantly
KOMSIYSKA D.

higher than that of patients with post-stroke depression.

Patients with post-stroke depression show mild typical depressive symptoms such as less depressive mood, anhedonia, disinterest, guilt, negative thoughts, depreciation, suicidal thoughts and anxiety.

In addition, somatic symptoms of depression do not lead to an increase to the diagnosing of depression in patients with stroke. Their results show that the clinical picture of post-stroke depression generally consists of symptoms with mild or moderate intensity, especially those considered as pillars for diagnosis of depression: depressed mood, loss of pleasure and lack of interest. Given the inaccuracy of the boundaries that separate the clinical forms of depression from subclinical and nonpathological forms, they situate post-stroke depression in the context of the biopsychosocial model in which the better understanding the psychopathological profile can provide timely diagnostics and therapeutic alternatives best suited to the difficult reality for patients with stroke (30).

\section{CONCLUSION}

Depressive disorder in the acute phase after a stroke was found in approximately $2 / 3(64.5 \%)$ of stroke patients, with predominantly mild and moderate depressive disorders with reduced mood, sadness, increased psychic and somatic anxiety, insomnia, somatic complaints and superfluous hypochondriac thoughts that can be derived from them.

\section{REFERENCES}

1. Verdelho, A., Hunon, H., Lebert, F., Pasquoer, F. and Leys, D., Depressive symptoms after stroke and relationship with dementia, Neurology, 62:905-911, 2004.

2. Hackett, M., Yapa, C., Parag, V. and Anderson, C., Frequency of depression after stroke. A systematic review of observational studies, Stroke, 36:13301340, 2005.

3. Tang, W., Chan, S., Chiu, H., Ungvari, G., Wong, K., Kwok, T., Mol, V., Wong, K., Richards, P. and Ahuja, A., Poststroke depression in Chinese patients: frequency, psychosocial, clinical, and radiological determinants, J Geriatr Psychiatry Neurol, 18(1):45-51, 2005.

4. Hadidi, N., Treat-Jacobson, D., Lindquist, R., Post-stroke depression and functional outcome: a critical review of literature, Heart and Lung, 38:151-162, 2009.

5. Andersen, G., Vestergaard, K., Riis, J. and Lauritzen, L., Incidence of post-stroke 
depression during the first year in a large unselected stroke population determined using a valid standardized rating scale, Acta Psychiatrica Scandinavica, 90:190-195, 1994.

6. Aben, I., Verhey, F., Strik, J., Lousberg, R., Lodder, J. and Honig, A., A comparative study into the one year cumulative incidence of depression after stroke and myocardial infarction, J of Neurol, Neurosurg and Psych, 74:581-585, 2003.

7. Paolucci, S., Gandolfo, C., Provinciali, L., Torta, R., Sommacal, S. and Toso, V., Quantification of the risk of poststroke depression: the Italian multicenter observational study DESTRO, Acta Psych Scandinavica, 112:272-278, 2005.

8. Morris, P., Robinson, R. and Raphael, B., Prevalence and course of depressive disorders in hospitalized stroke patients, Intern $J$ of Psych in Med, 20:349-364, 1990.

9. Estrom, M., Adolfsson, R. and Asplund, K., Major depression in stroke patients: a 3-year longitudinal study, Stroke, 24:976-982, 1993.

10. Mihaylova, B., Petkova, I., RankovaYotova, Ch., Dimitrova, G., Tanev, I., Ivanova, S., Tsakova, A., Svinarov, D. Plasma endothelin-1 and endothelin-A receptor concentrations in patients with primary open-angle glaucoma. Biotechnol \& Biotechnolog Eq, 31(4):782-787, 2017.

11.Dakov, N., Kostova, St., Tanev, Iv. Selective laser trabeculoplasty in primary open angle glaucoma - efficiency and correlated parameters. Compt rend del Acad Bulg des Sci 71(2):288-298, 2018.

12.Komsiiska, D. Oxidative stress and stroke: a review of upstream and downstream antioxidant therapeutic options. Comp Clin Path, 1-12, 2019.

13. Burvill, P., Johnson, G., Jamrozik, K., Anderson, C., Stewart-Wynne, E. and Chakera, T., Prevalence of depression after stroke: The Perth Community Stroke Study, The British J of Psych, 166:320-327, 1995.

14. Komsiyska, D., Oxydative stress and poststroke depression. $\operatorname{Tr} J$ Sci, 3: 249-253, 2018.

15.Komsiyska, D., Petkov Y. Post-stroke depression and anxiety, $\operatorname{Tr} J$ Sci 1: 56-63, 2019.

16. Robinson, R., Poststroke depression: prevalence, diagnosis, treatment, and desease progression, Biol Psychiatry, 54(3):376-387, 2003.

17. Komsiyska, D., Penev, M., Petrov, Y., Hristova, M. The role of anxiolitics in the treatment of patient with arterial hypertension accompanied by anxiety disorders, $\operatorname{Tr} J \mathrm{Sci}$, 13 (Suppl 2):133-135, 2015.
18. Komsiyska D., Petkov Y. The role of oxidative stress in the etiopathogenesis of depression $\operatorname{Tr} J$ Sci.,1: 81-93, 2019.

19. Fedoroff, J., Starkstein, S., Parikh, R., Price, T. and Robinson, R., Are depressive symptoms nonspecific in patients with acute stroke?, The American J of Psych, 148:11721176, 1991.

20. Gainotti, G., Azzoni, A. and Marra, C., Frequency, phenomenology and anatomical clinical correlates of major post-stroke depression, The British Journal of Psychiatry, 175:163-167, 1999.

21. Tang, W., Caeiro, L., Lau, C., Liang, H., Mok, V., Ungvari, G. and Wong, K., Apathy and suicide-related ideation 3 months after stroke: a cross-sectional study, BMC Neurol, 15:60, 2015.

22. Kishi, Y., Robinson, R. and Kosier, J., Suicidal plans in patients with stroke: comparison between acute-onset and delayedonset suicidal plans, Int Psychogeriatr, 8(4):623-634, 1996.

23. Eriksson, M., Glader, E., Norrving, B. and Asplund, K., Poststroke suicide attempts and completed suicides: a socioeconomic and nationwide perspective, Neurology, 84(17):1732-1738, 2015.

24. Garden, F., Garrison, S. and Jain, A., Assessing suicide risk in stroke patients: review of two cases, Arch Phys Med Rehabil, 71(12):1003-1005, 1990.

25.Lipsey, J., Robinson, R., Pearlson, G., Rao, K. and Price, T., Nortriptyline treatment of post-stroke depression. A double-blind study, Lancet, 1(8372):297-300, 1984.

26. Savina, M., Post-stroke mental violations: clinical, statistical, pathogenetic, prognostic aspects, Dissertation, pp. 283-284, 311, 2016.

27. Arnaudova, M., Introduction to the advanced psychiatry and the late age, University Publishing House of the Medical University Varna, Varna, 260-262, 302, 2015.

28. Rabi-Zikic, T., Nedic, A., Zarkov, M., Slankamenac, P., Dobrenov, D. and Zivanovic, Z., Poststroke depression: diagnosis of depression, phenomenology and specificity of depressive symptoms, Med Pregl, 62(3-4):148-152, 2009.

29. Starchina, Y., Parfenov, V., Chazova, I. and Sinitsyn, V., Cognitive functions and emotional status of patients with stroke in regard to antihypertensive therapy, Neurol and Psychiatrist Journal, 15:39-44, 2005.

30. da Rocha e Silva, C., Alves Brasil, M., Matos do Nascimento, E., de Braganca Pereira, B. and Andre, C., Is poststroke depression a major depression?, Cerebrovasc Dis, 35(4):385-391, 2013. 